\title{
Relationship of Pruritus with Biochemical and Haematological Parameters in Haemodialysis Patients (A Single Center Study).
}

\author{
Ban Ghassan* \\ Jaffar N. Jaffar Alalsaidissa** \\ Ali J. Hashim Al- Saedi**** \\ Sabah N. Alawchi $* * * *$
}

\author{
BSc, MSc \\ MBChB, MSc, PhD \\ $\mathrm{MBChB}, \mathbf{P h D}$
}

\author{
BSc, PhD
}

\begin{abstract}
:
Background: Pruritus often constitutes a major problem for patients with end stage renal disease. The pathophysiological mechanism of chronic kidney disease -associated pruritus is poorly defined.

Objectives: To evaluate the prevalence of uremic pruritus in dialysis patients and their correlation with the laboratory and clinical parameters in some Iraqi patients with chronic renal disease.

Fac Med Baghdad 2015; Vol.57, No.4 Received:April,2015 Accepted:Sept, 2015 hemodialysis. Blood urea, creatinine, calcium, phosphorus, alkaline phosphatase and parathyroid hormone parathyroid hormone were determined. Complete blood count was also performed.

Results: Of the 103 patients included in the study the, 79 patients (76.7\%) had pruritus of whom, $27(34.1 \%)$ had mild pruritus, $30(38 \%)$ had moderate and $22(27.9 \%)$ had severe pruritus. No significant association $(\mathrm{p}>0.05)$ was found between pruritus and each of age and haemodialysis duration. There was no significant difference $(\mathrm{p}>0.05)$ in the urea, creatinine, calcium, phosphorus, alkaline phosphatase, parathyroid hormone, hemoglobin, hematocrit, white blood cells, neutrophils, eoisinophils, basophils mean values among patients who had no pruritus, mild, moderate and severe pruritus. A significant positive correlation $(\mathrm{p}>0.05)$ was found between severity of pruritus in pruritic patients and each of age, hemoglobin and hematocrit levels.

Conclusion: Our study showed that age, hemoglobin and hematocrit levels had a significant positive correlation with the severity of pruritus in pruritic patients.

Keywords: pruritus, haemodialysis, biochemical and haematological parameters.
\end{abstract}

\section{Introduction:}

Pruritus often constitutes a major problem for patients with end stage renal disease (ESRD) 1 and it is the most important skin manifestation during dialysis.2 According to most sources, more than half of patients undergoing haemodialysis (HD) complain of varying degrees of pruritus. 1 The intensity can be mild, moderate or severe with sleep disorders, discomfort and increased irritability, disturbance of daily activity or general stress. Pruritus may be acute or chronic, localized or generalized.3 Many factors have been considered in the pathophysiological process of chronic kidney disease (CKD)-associated pruritus, including xerosis, abnormalities in afferent pain fibers, cutaneous divalent ion content, alterations in number of skin mast cells, inadequate removal of middlemolecular- weight uremic toxins and allergic sensitization 4 hypervitaminosis A, cytokines, nitric oxide, creatinine, total protein, urea, ferritin, and transferrin disorders.5 Other

* College of Science, University of Baghdad.

** College of Medicine, University of Baghdad.

*** Corresponding author: Ali Jasim Hashim Al-Saedi Nephrology and transplant center./medical city complex.

Email:dralijasm.hashim@yahoo.com

Nephrologyand Transplant Center.

**** Dept. of Biology, College of Science, University of Baghdad. factors contribute to uremic pruritus (UP) such as: secondary hyperparathyroidism (SHPT), hyperphoshatemia, inadequate dialysis, increased $\beta 2$-microglobulin levels, high aluminum levels, immune dysfunction 6 and changes in the opioidergic system, iron deficiency anaemia and inflammation.7 Additional factors implicated include high serum levels of magnesium, bile acids, histamine, and substance P.4 Various proposed treatments have resulted in limited success in providing longterm relief from pruritus in HD patients. 8

The main objectives of this study are to evaluate the prevalence of UP in HD patients and their correlation with the laboratory and clinical parameters.

\section{Patients and methods:}

This descriptive-analytic, cross-sectional study was conducted on 103 patients who were on HD at Alkindy teaching hospital in Iraq from February 2014 to May 2014. All the patients were treated by conventional HD 4-5 hours, three times a week. Polysulphone membranes were used as dialyser, a dialysate was produced from $140 \mathrm{mmol} / \mathrm{L}$ sodium, $2 \mathrm{mmol} / \mathrm{L}$ potassium, $1.5 \mathrm{mmol} / \mathrm{L}$ calcium, $0.5 \mathrm{mmol} / \mathrm{L}$ magnesium, $111 \mathrm{mmol} / \mathrm{L}$ chloride, $3 \mathrm{mmol} / \mathrm{L}$ acetate and $32 \mathrm{mmol} / \mathrm{L}$ bicarbonate. Information related to the patients, including age, 
gender, duration on dialysis, pruritus period, pruritus severity, pruritus distribution, pruritus frequency, effect of pruritus on sleeping and waking up were extracted from questionnaires and medical reports.

Pruritus Measurement: To study pruritus that might occur in chronic HD patients, the patients were questioned, if they had pruritus. Severity of pruritus was assessed by a method based on the one proposed by Layegh et al.9

The questionnaire included the items below:

1- Pruritus period: one point for each time of the day (morning, afternoon, and night). If the patient suffers from the pruritus all day, give 3 points.

2- Pruritus severity: the severity of pruritus was assessed subjectively and scored as follows: pruritus without the need to scrape (1), limited need to scrape (2), consistent need to scrape (3), scrape without improving (4), and irritant pruritus (5).

3- Pruritus distribution: limited to one or two regions for example arm, leg, or trunk (1), generalized pruritus (5).

4- Pruritus frequency and duration: every two short episodes ( $<10 \mathrm{~min}$ ) or one long episode $(\geq 10 \mathrm{~min}$ ) received 1 point, with a maximum of 5 points for ten or more short episodes or for five or more long episodes.

5- Sleeping: the sleeping effect of pruritus was measured by asking individuals whether their pruritus interfered partially, completely, or not at all with certain sleep hours: no night sleep because of pruritus (10) sleep less than 7 hours.

6- Waking up: each wake up (1) and wake up more than 5 times (5).

For severity, distribution and frequency, separate scores were recorded for the morning and afternoon. Thus, as Table 1 shows, the highest possible score for a 24-hour period was 48 points.

Based on this pointing system, pruritus was categorized as mild: 1 to 16 ; moderate: 17 to 32 ; severe: 33 to 48 .
Table 1: Pruritus-pointing system.

\begin{tabular}{ccccc}
\hline Item & Morning & Afternoon & Night & Total \\
\hline Period & 1 & 1 & 1 & 3 \\
\hline Severity & 5 & 5 & - & 10 \\
\hline Distribution & 5 & 5 & - & 10 \\
\hline Frequency & 5 & 5 & - & 10 \\
\hline Sleeping & - & - & 10 & 10 \\
\hline Waking up & - & - & 5 & 5 \\
\hline Total & 16 & 16 & 16 & 48 \\
\hline
\end{tabular}

Biochemistry and haematology: Venous blood sample was collected in the morning immediately before dialysis sessions. The serum was obtained after centrifugation and serum parathyroid hormone was measured by using PTH intact ELISA kit. Urea, creatinine, serum calcium, serum phosphorus, serum total alkaline phosphatase were measured by standard laboratory techniques. Venous blood sampling was collected using tubes containing EDTA and complete blood count was performed by an automated analyzer (Abbott auto analyzer for haematology).

Statistical Analysis: Statistical package for social sciences version 20 (SPSS 20) was used for data analysis. Findings with $\mathrm{P}$ value less than 0.05 were considered significant.

\section{Results:}

Of the 103 patients included in the study, 79 patients $(76.7 \%)$ had pruritus of whom, $27(34.1 \%)$ had mild pruritus, 30 $(38 \%)$ had moderate and $22(27.9 \%)$ had severe pruritus. The remaining 24 (23.3\%) cases had never suffered from pruritus. No significant association $(p>0.05)$ was found between each of age $\left(\chi^{2}=11.265, \mathrm{p}=0.081\right)$, duration of HD $\left(\chi^{2}=6.660\right.$, $\mathrm{p}=0.084$ ) and pruritus (Table 2).

Table 2: Distribution of study sample according to different degrees of pruritus and to personal and haemodialysis factors.

\begin{tabular}{|c|c|c|c|c|c|c|}
\hline \multirow{3}{*}{ Variables } & \multicolumn{4}{|c|}{ Pruritus } & \multirow{3}{*}{$\chi^{2}$} & \multirow{3}{*}{ Pvalue } \\
\hline & No pruritus & Mild & Moderate & Severe & & \\
\hline & $\mathrm{N}=24(100 \%)$ & $\mathrm{N}=27(100 \%)$ & $\mathrm{N}=30(100 \%)$ & $\mathrm{N}=22(100 \%)$ & & \\
\hline \multicolumn{7}{|l|}{ Age group } \\
\hline$>46$ & $8(33.3 \%)$ & $15(55.6 \%)$ & $11(36.7 \%)$ & $6(27.3 \%)$ & \multirow{3}{*}{11.265} & \multirow{3}{*}{0.081} \\
\hline $46-65$ & $10(41.7 \%)$ & $11(40.7 \%)$ & $16(53.3 \%)$ & $9(40.9 \%)$ & & \\
\hline$>65$ & $6(25.0 \%)$ & $1(3.7 \%)$ & $3(10.0 \%)$ & $7(31.8 \%)$ & & \\
\hline \multicolumn{7}{|c|}{ Duration of HD } \\
\hline$>1$ year & $15(62.5 \%)$ & $11(40.7 \%)$ & $16(53.3 \%)$ & $6(27.3 \%)$ & \multirow{2}{*}{6.660} & \multirow{2}{*}{0.084} \\
\hline$\geq 1$ year & $9(37.5 \%)$ & $16(59.3 \%)$ & $14(46.7 \%)$ & $16(72.7 \%)$ & & \\
\hline
\end{tabular}

The results of biochemical tests showed no significant differences $(p=0.05)$ in the urea $(p=0.177)$, creatinine $(p=0.337), P T H$ $(\mathrm{p}=0.224)$, total ALP $(\mathrm{p}=0.500)$, serum calcium $(\mathrm{p}=0.390)$ and phosphorus $(\mathrm{p}=0.873)$ levelsamong patients with no pruritus, mild, moderate and severe pruritus (Table 3$)$. No significant variations $(\mathrm{p}=0.05)$ in the mean of $\mathrm{Hb}(\mathrm{p}=0.057)$ and $\mathrm{Hct}$ 
$(\mathrm{p}=0.069)$ levels were found among patients who had no pruritus, mild, moderate and severe pruritus. No significant difference $(p=0.05)$ was existed in the averages of $\operatorname{WBC}(p=0.194)$, neutrophil $(p=0.997)$, eosinophil $(p=0.173)$ and basophil $(p=0.706)$ counts among the four groups.

Table 3: descriptive statistics for biochemical variables, serum electrolytes and haematological findings in patients with different degrees of pruritus.

\begin{tabular}{|c|c|c|c|c|c|}
\hline \multirow{3}{*}{ Variables } & \multicolumn{4}{|c|}{ Pruritus } & \multirow{3}{*}{ P value } \\
\hline & $\begin{array}{c}\text { No pruritus } \\
\mathbf{N}=\mathbf{2 4}\end{array}$ & $\begin{array}{l}\text { Mild } \\
\text { N=27 }\end{array}$ & $\begin{array}{c}\text { Moderate } \\
\mathbf{N}=\mathbf{3 0}\end{array}$ & $\begin{array}{l}\text { Severe } \\
\mathbf{N}=22\end{array}$ & \\
\hline & Mean \pm SD & Mean \pm SD & Mean \pm SD & Mean \pm SD & \\
\hline Blood urea (mg/dl) & $157.6 \pm 56.2$ & $160.4 \pm 49.7$ & $150.1 \pm 42.9$ & $183.2 \pm 68.2$ & 0.177 \\
\hline Serum creatinine(mg/dl) & $5.1 \pm 1.5$ & $4.7 \pm 1.3$ & $4.4 \pm 1.9$ & $5.1 \pm 1.9$ & 0.337 \\
\hline PTH (pg/ml) & $403.6 \pm 383.9$ & $386.6 \pm 424.0$ & $268.3 \pm 249.8$ & $238.5 \pm 329.8$ & 0.224 \\
\hline $\operatorname{ALP}(\mathbf{U} / \mathbf{L})$ & $161.7 \pm 114.0$ & $167.0 \pm 133.2$ & $181.1 \pm 224.3$ & $183.0 \pm 176.7$ & 0.500 \\
\hline Calcium (mg/dl) & $8.3 \pm 1.4$ & $9.0 \pm 1.8$ & $9.0 \pm 1.8$ & $8.8 \pm 1.3$ & 0.390 \\
\hline Phosphorus (mg/dl) & $7.0 \pm 2.3$ & $6.7 \pm 1.8$ & $6.7 \pm 2.4$ & $6.5 \pm 2.0$ & 0.873 \\
\hline $\mathrm{Hb}(\mathrm{g} / \mathrm{dl})$ & $8.7 \pm 2.7$ & $7.7 \pm 1.6$ & $9.0 \pm 1.9$ & $9.0 \pm 1.9$ & 0.057 \\
\hline Hematocrit (\%) & $29.0 \pm 9.4$ & $25.4 \pm 5.3$ & $29.6 \pm 6.3$ & $30.0 \pm 6.0$ & 0.069 \\
\hline 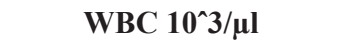 & $6.6 \pm 2.3$ & $7.0 \pm 2.8$ & $7.1 \pm 2.6$ & $8.4 \pm 4.1$ & 0.194 \\
\hline Neutrophil (\%) & $65.8 \pm 9.9$ & $65.5 \pm 9.3$ & $65.2 \pm 8.7$ & $65.4 \pm 10.9$ & 0.997 \\
\hline Eosinophil (\%) & $3.0 \pm 3.1$ & $3.3 \pm 2.3$ & $3.2 \pm 2.0$ & $4.1 \pm 2.5$ & 0.173 \\
\hline Basophil (\%) & $1.9 \pm 0.6$ & $1.9 \pm 0.7$ & $1.9 \pm 0.6$ & $1.7 \pm 0.7$ & 0.706 \\
\hline
\end{tabular}

Correlation of severity of pruritus with study variable in pruritic patients: A significant positive correlation $(\mathrm{p}>0.05)$ was found between severity of pruritus in pruritic patients and age $(r=0.288, p=0.010)$. Similarly a significant positive correlation $(p>0.05)$ was found between severity of pruritus and each of Hb level $(\mathrm{r}=0.283, \mathrm{p}=0.011)$ and Hct level $(\mathrm{r}=$ $0.296, p=0.008$ ) (table 4 ). There was no significant correlation $(p>0.05)$ between severity of pruritus in pruritic patients and duration of HD $(r=0.181, p=0.111)$, blood urea $(r=0.114$, $\mathrm{p}=0.317)$, serum creatinine $(\mathrm{r}=0.062, \mathrm{p}=0.587)$, PTH $(\mathrm{r}$ $=-0.160, \mathrm{p}=0.159), \operatorname{ALP}(\mathrm{r}=0.068, \mathrm{p}=0.549)$, calcium $(\mathrm{r}=$ -0.035, $\mathrm{p}=0.757)$ and phosphorus $(\mathrm{r}=-0.087, \mathrm{p}=0.447)$.No significant correlation $(\mathrm{p}>0.05)$ was also observed between severity of pruritus and some haematological findings such as WBCs $\quad(r=0.199, p=0.078)$, nutrophil $(r=0.027, p=0.813)$, eosinophil $(\mathrm{r}=0.129, \mathrm{p}=0.257)$ and basophil counts $(\mathrm{r}=$ $-0.024, p=0.830$ ).
Table 4: Correlation of severity of pruritus with different study variables in pruritic patients.

\begin{tabular}{|c|c|c|}
\hline \multirow{2}{*}{ Variables } & \multicolumn{2}{|c|}{ Spearman's rho } \\
\hline & Correlation coefficient & P value \\
\hline Age (year) & 0.288 & $0.010 *$ \\
\hline Duration of HD & 0.181 & 0.111 \\
\hline Blood urea (mg/dl) & 0.114 & 0.317 \\
\hline Serum creatinine $(\mathrm{mg} / \mathrm{dl})$ & 0.062 & 0.587 \\
\hline PTH (pg/ml) & -0.160 & 0.159 \\
\hline $\operatorname{ALP}(\mathbf{U} / \mathbf{L})$ & 0.068 & 0.549 \\
\hline calcium (mg/dl) & -0.035 & 0.757 \\
\hline phosphorus (mg/dl) & -0.087 & 0.447 \\
\hline $\mathrm{Hb}(\mathrm{g} / \mathrm{dl})$ & 0.283 & $0.011 *$ \\
\hline Hematocrit (\%) & 0.296 & $0.008 *$ \\
\hline WBC $10^{\wedge} 3 / \mu \mathrm{l}$ & 0.199 & 0.078 \\
\hline Neutrophils (\%) & 0.027 & 0.813 \\
\hline Eosinophils (\%) & 0.129 & 0.257 \\
\hline Basophils (\%) & -0.024 & 0.830 \\
\hline
\end{tabular}

$* \mathrm{P}$ value $>0.05$, significant correlation. 


\section{Discussion:}

The incidence of uremic itch varies between studies and depends on patients included for evaluation.10 In this study, pruritus was found in $76.7 \%$ of the patients of whom, (34.1\%) had mild pruritus, $(38 \%)$ had moderate and $(27.9 \%)$ had severe pruritus. The study of Resic et al.6 showed that pruritus was found in 45 patients $(58.44 \%)$, severe in $17.78 \%$, moderate in $40.0 \%$ and mild in $42.2 \%$ of the patients. These differences may be due to the prevailing climatic conditions,(11) moreover, it is worth mentioning that a major drawback for studying and comparing results from different studies is the lack of a uniform way for assessment of this very subjective symptom. 1 In this study no significant association was found between pruritus and HD duration consistent with the study of Akhyani et al.(1) No significant association was also found between age and pruritus in this study consistent with another study of Tajbakhsh.(11) In present study, both urea and serum creatinine had no significant differences among the patients who had no pruritus, mild, moderate and severe pruritus similar to the studies of Virga et al.,12 Chiu et al.,13 and Chen et al.(14)

In the current study, there was no statistically significant differences in PTH level between patients who had no pruritus, mild, moderate and sever pruritus similar to the studies of Chiu et al.13 and Razeghi et al(.15) The results of this study demonstrated that no significant difference was existed in the level of both serum calcium and phosphorus among patients who had no pruritus, mild, moderate and severe pruritus similar to the studies of Chiu et al.,13 Chen et al.14 and Razeghi et al.(15 )According to the findings of the present study, no significant variations in the mean of $\mathrm{Hb}$ was found among patients who had no pruritus, mild, moderate and severe pruritus consistent with other studies of Chen et al.14 and Razeghi et al.(15)

In this study, as in the study of Virga et al.12 no significant variation in the mean of Hct level was found among patients of four groups. White blood cell counts are increased in patients with UP 16-17 but most patients who were included in this study were associated with normal WBC counts as well as no significant difference in WBC count was found among the patients who had no pruritus, mild, moderate and severe pruritus similar to the results of Chiu et al.(13) Similar to the result of present study, Pisoni et al.7 indicated that no significant relationship was seen between pruritus and neutrophil count or neutrophil percentage. Present study explained that the averages of neutrophil count were insignificantly different among patients of four groups. Kojima et al.18 demonstrated that eosinophilia may have been induced by an allergic reaction to HD related materials and the resultant increase in eosinophil might cause allergic symptoms such as itching. But the result of present study revealed that eosinophil count was within the normal range in most patients who included in the study and non-existence of statistically significant different in the averages of eosinophil count among the patients who had no pruritus, mild, moderate and severe pruritus. In this study the averages of basophil counts were insignificantly different among patients who had no pruritus, mild, moderate and severe pruritus. This finding agrees with the data obtained by Diehn and Tefferi19 which indicated that no significant correlation was found between active pruritus and basophils count. With respect to the correlation of pruritus severity with study variable in pruritic patients:

In the present study, there was no significant correlation between severity of pruritus in pruritic patients and duration of HD. In contrast, the result of Narita et al.20 revealed that the group with severe pruritus had a significantly longer duration of dialysis. Present study found a significant positive correlation between severity of pruritus in pruritic patients and age. This result disagrees with result of Narita et al.20 It may be explained to more skin xerosis in older patients in this study. Many studies indicated that aging skin is susceptible to pruritic disorders because of the cumulative effects that the environment has on the skin and because of changes to the skin structure that occur as individual gets older.(21) The result of Narita et al.20 revealed that the group with severe pruritus had a significantly higher levels of serum creatinine and blood urea. In the present study, there was no significant correlation between severity of pruritus in pruritic patients and both of blood urea and creatinine. Other factors may have attributable role such as pruritogenic middle-molecular weight molecules 20 that accumulate in the dialysis patient because they are poorly dialyzable as a result of their molecular size. (22) In a study by Narita et al.20 the group with severe pruritus had a significantly higher level of intact PTH. In the present study, no significant correlation was observed between the severity of pruritus and PTH. In this study the level of PTH in sever pruritic patients was lower than those with moderate and mild pruritus and this may explain our result. Narita et al.20 in their study showed that the group with severe pruritus had a significantly higher level of serum calcium and phosphorus. In this study no correlation was found between each of calcium and phosphorus levels and severity of pruritus in pruritic patients. Our result may be because we have few patients with calcium level higher than normal and it seem that the average level of phosphate in patients with severe pruritus is not differ from those with mild and moderate pruritus. These data suggest that raised phosphorus levels is not the cause of severity of itching. We found a significant positive correlation between severity of pruritus in pruritic patients and each of 
$\mathrm{Hb}$ level and Hct level. Our result is supported by Snit et al.23 who found that higher $\mathrm{Hb}$ was related to higher risk of itching and its intensity. Further studies required for the types of anemia and iron indices to proof this result. No significant correlation was also observed between severity of pruritus in pruritic patients and both of WBC and neutrophil counts. This may be attributed to the fact that WBC and neutrophil counts were within the normal range in most patients involved in this study. Kang et al.24 revealed that there was a positive correlation between the prevalence of eosinophilia and severity of pruritus in patients on HD. Normal eosinophil count in most patients who were included in present study may explain this result which revealed that no significant correlation was observed between severity of pruritus and eosinophil count. No significant correlation was found between the severity of pruritus in pruritic patients and basophil counts. Other factors may have attributable role such as histamine which is mainly released by skin mast cells and basophils. 25 Other researchers 26 reported that itching was correlated well with the numbers of skin mast cells but not with circulating basophils or whole blood histamine. We did not measure level of histamine and skin mast cells in our patients.

\section{Conclusion:}

Our study shows that the severity of pruritus in pruritic patients positively correlated with age, hemoglobin and hematocrit levels.

\section{Author contributions:}

Ban Ghassan: performing the practical steps and writing of the research.

Dr. Jaffar Nouri Jaffar Alsaidissa: reviewing the research scientifically and linguistically.

Dr. Ali J. Hashim Al- Saedi: helping in the planning and guidance throughout the research.

Dr. Sabah AL Alawchi: assistance in scientific advices.

\section{References:}

1-Akhyani M, Ganji M-R, Samadi N, Khamesan B, Daneshpazhooh M.. Pruritus in hemodialysis patients. BMC Dermatology 2005; 5:7. [IVSL]

2-Avermaete A, Altmeyer P, Bacharach $\square$ Buhles M. Skin changes in dialysis patients: a review. Nephrol. Dial. Transplant 2001; 16 (12): 2293-2296.

3-Yonova D. Pruritus in certain internal diseases. Hippokratia. 2007;11(2): 67-71.

4- Patel T S, Freedman B I, Yosipovitch G. An Update on Pruritus Associated With CKD. Am J Kidney Dis 2007; 50:1120.
5- Welter $E$ de $Q$, Frainer $R H$, Maldotti A, Losekann A, Weber M B. Evaluating the association between alterations in mineral metabolism and pruritus in hemodialysis patients. An Bras Dermatol 2011; 86(1):31-6.

6- Resic H, Alendar F, Kukavica N, Masnic F, Cengic B. Uremic Pruritus in Haemodialysis Patients. BANTAO Journal 2007; 5 (1): 19.

7- Pisoni R L, Wikstrom B, Elder S J, Akizawa T, Asano Y, Keen M L, Saran R, Mendelssohn D C, Young E W, Port $F$ K. Pruritus in haemodialysis patients: international results from the Dialysis Outcomes and Practice Patterns Study (DOPPS). Nephrol Dial Transplant 2006; 21(12): 3495-505.

8- Hsu M M, Yang C C. Uraemic pruritus responsive to broadband ultraviolet (UV) B therapy does not readily respond to narrowband UVB therapy. Br J Dermatol 2003;149: 888889.

9- Layegh P, Mojahedi M J, Malekshah P E T, Pezeshkpour F, Vahedian M, Nazemian F, Pour F S. Effect of oral granisetron in uremic pruritus. Indian J Dermatol Venereol Leprol 2007; 73 (4): $231-234$.

10- Szepietowski J C, Sikora M, Kusztal M, Salomon J, Magott M, Szepietowski T. Uremic Pruritus: A Clinical Study of Maintenance Hemodialysis Patients. The Journal of Dermatology 2002; 29: 621-627.

11- Tajbakhsh R, Joshaghani H R, Bayzayi F, Haddad $M$, Qorbani M. Association between pruritus and serum concentrations of parathormone, calcium and phosphorus in hemodialysis patients. Saudi Journal of kidney dialysis and transplantation 2013. 24 (4): 702-706.

12-Virga G, Visentin I, La Milia V, Bonadonna A. Inflammation and pruritus in hemodialysis patients. Nephrol Dial Transplant 2002; 17:2164-2169.

13- Chiu Y-L, Chen H-Y, Chuang Y-F, Hsu S-P, Lail C-F, Pail $M-F$, Yang S-Y, Peng Y-S. Association of uraemic pruritus with inflammation and hepatitis infection in haemodialysis patients. Nephrol Dial Transplant 2008; 23: 3685-3689.

14- Chen H-Y, Chiu Y-L, Hsu S-P, Pai M-F, Lai C-F, Yang J-Y, Peng Y-S, Tsai T-J, Wu K-D. Elevated C-reactive protein level in hemodialysis patients with moderate/severe uremic pruritus: a potential mediator of high overall mortality. Q J Med 2010. doi:10.1093/qjmed/hcq036.

15-Razeghi E, Tavakolizadeh S, Ahmadi F. Inflammation and Pruritus in Hemodialysis Patients. Saudi Journal of kidney dialysis and transplantation 2008; 19 (1): 62-66.

16-Asicioglu E, Kahveci A, Koç M, Ozener C. Uremic Pruritus: Still Itching. Turk Neph Dial Transpl 2011; 20 (1): 7-13.

17- Kimmel M, Alscher D M, Dunst R, Braun N, Machleidt C, Kiefer T, Stulten C, van der Kuip H, Pauli-Magnus C, Raub U, Kuhlmann U, Mettang T. The role of micro-inflammation in the 
pathogenesis of uraemic pruritus in haemodialysis patients. Nephrol Dial Transplant 2006; 21: 749-755.

18- Kojima K, Oda K, Homma H, Takahashi K, Kanda Y, Inokami T, Uchida S. Effect of vitamin E-bonded dialyzer on eosinophilia in haemodialysis patients. Nephrol Dial Transplant 2005; 20: 1932-1935.

19- Diehn F, Tefferi A. Pruritus in polycythaemia vera: prevalence, laboratory correlates and management. British Journal of Haematology 2001; 115 (3): 619-621. [IVSL]

20- Narita I, Alchi B, Omori K, Sato F, Ajiro J, Saga D, Kondo D, Skatsume M, Maruyama S, Kazama JJ, Akazawa K, Gejyo F. Etiology and prognostic significance of severe uremic pruritus in chronic hemodialysis patients. Kidney International 2006; 69: 1626-1632.

21- Grundman S, Stander S. Evaluation of chronic pruritus in older patients. Aging Health 2010; 6:53-66.

22- Kfoury L W, Jurdi M A. Uremic pruritus. JNEPHROL 2012; 25(05): 644-652.

23- Snit M, Gawlik R, Lacka-Gazdzik B, Kuzniewicz R, Dwornicki M, Owczarek A, Grabiec M W P, Grzeszczak W. Substance $P$ and intensity of pruritus in hemodialysis and peritoneal dialysis patients. Med Sci Monit 2013; 19: 723732.

24- Kang K Y, You M Y, Lee J S, Ihm C W, Moon C Y, Park S K. Relationship between Pruritus and Eosinophilia in Patients on Hemodialysis. Korean J Nephrol 1999. 18(4):585-591.

25- Narita I, Iguchi S, Omori K, Gejyo F. Uremic pruritus in chronic hemodialysis patients. J Nephrol 2008; 21:161-5.

26- Jackson N, Burt N, Crocker J, Boughton B. Skin mast cells in polycythaemia vera relationship to the pathogenesis and treatment of pruritus. British Journal of Dermatology 1987; $116(1): 21-29$. 